\title{
Synthetic Bone Graft versus Autograft Obtained from the Spinous Process in Posterior Lumbar Interbody Fusion
}

\author{
Tayfun CAKIR ${ }^{1}$, Coskun YOLAS ${ }^{2}$ \\ ${ }^{1}$ Erzincan Binali Yildirim University, Faculty of Medicine, Department of Neurosurgery, Erzincan, Turkey \\ ${ }^{2}$ Health Sciences University, Erzurum Regional Training and Research Hospital, Department of Neurosurgery, Erzurum, Turkey \\ Corresponding author: Tayfun CAKIR tayfuncakir198375@gmail.com
}

\section{ABSTRACT}

AIM: To compare the clinical and radiological results of using synthetic graft versus spinous process lumbar interbody graft obtained intraoperatively.

MATERIAL and METHODS: This retrospective study included 102 patients with degenerative lumbar spondylolisthesis who underwent one-segment posterior lumbar interbody fusion (PLIF) followed by one-segment posterior transpedicular instrumentation. PLIF surgery was performed using a local solid bone graft obtained from the spinous process in group A and using a synthetic solid calcium hydroxyapatite block in group B. The Oswestry Disability Index (ODI), visual analogue scale (VAS) scores, degree of bone formation, intervertebral disc heights at the operation segment, wound infection, and instrumentation complications were compared between the 6-month and 5-year follow-ups.

RESULTS: In both groups, ODI and VAS scores significantly improved at the 6-month and 5-year follow-up. Bone formation at both 6 months and 5 years were higher in group A than that in group B, but without a significant difference when compared. Moreover, the difference in maintaining the intervertebral disc heights was not significant between the two groups. Surgical wound infection more commonly occurred in group B, but without significant difference between the two groups, and rod fractures were observed in two patients in group B; however, no metal breakage was observed in group A.

CONCLUSION: Successful fusion of the intervertebral space and intervertebral height restoration can be achieved and maintained with an autograft from the patient's spinous processes.

KEYWORDS: Lumbar spondylolisthesis, Spinous process, Autograft, PLIF

ABBREVIATIONS: PLIF: Posterior lumbar interbody fusion, BMI: Body mass index, ODI: Oswestry disability index, VAS: Visual analog scale, MIC: Maximal information coefficient

\section{INTRODUCTION}

$\mathrm{I}$ ncidences of degenerative disc disease and spondylolisthesis have been gradually increasing in the society (2). Unbalanced loading and neural structure compressionin these patients are caused by mechanical and neurological symptoms. When conservative treatments inadequately relieve painor deteriorated neurological function occur, surgery may be considered to decompress the neural structures and stabilize the spine $(13,16,17)$. Posterior transpedicular instrumented fusion has been performed as one of the most common surgical procedures for the treatment of spondyIolisthesis $(2,24,25,27,29)$. In addition to the transpedicular instrumented fusion, posterior lumbar interbody fusion (PLIF) first described by Cloward in 1940 has been safely performed to date $(7,8,12)$. As known degenerative spondylolisthesis begins with intervertebral disc dehydration with decreased tensile strength of the annulus fibrosus, a corresponding loss of the intervertebral disc height may follow. This may lead to the posterior facet joint subluxation and an abnormal motion 
pattern that causes segmental instability $(4,20)$. Therefore, the PLIF technique is theoretically advantageous by increasing the segmental stability, restoring intervertebral disc height, decompressing nerve roots, and reinforcing the weight-bearing axis along the anterior segment of the spinal column $(7,11)$. With the increased use of this surgical technique, the demand for an optimal bone graft for the intervertebral space has continuously increased $(18,26)$. Normally, intervertebral cages or the bone graft obtained from the iliac crest has been safely and frequently used for years $(11,18,26)$. As has been known, intervertebral cages consisted of expensive materials and are associated with severaldisadvantages. Similarly, obtaining a bone graft from the iliac crest hasalsoseveral disadvantages,such as increased operation time, blood loss, and postoperative donor site pain (15). Thus, bone fragments obtained from the spinous process intraoperatively were placed only into the intervertebral space, confirming the hypothesis that enough fusion can be achieved without the need for any cage. Some previous studies showed that PLIF surgery using a natural cage made from the patient's spinous process can achieve a satisfactory interbody fusion, sufficient balance, greater mechanical strength, larger contact area, and better bilateral disc height restoration (14). Therefore, we describe our experience with this technique by comparing the methods using a synthetic calcium hydroxyapatite block as an interbody graft in this study.

\section{MATERIAL and METHODS}

\section{Patients}

This retrospective study included 102 patients with degenerative lumbar spondylolisthesis who underwent one-segment PLIF, followed by one-segment posterior transpedicular instrumented fusion performed by a single surgeon (CY) between 2010 and 2014. To validate the degenerative lumbar spondylolisthesis diagnosis, all patients underwent magnetic resonance imaging within 6 months preoperatively and were managed with conservative treatments, such as physical therapy (passive modalities and aerobic exercises) and anti-inflammatory drugs for at least 3 months. However, surgical treatment was recommended for patients who did not respond to these treatments. History of lumbar disc surgery, vertebral fracture, any signs of infection, tumors or malignancies, and drug or alcohol use were set as exclusion criteria. The degree of spondylolisthesis in all patients was grade 1 or 2 according to the Meyerding classification.

Group A: Clinical and radiological data of all patients who underwent PLIF using bone fragments obtained from the patient's spinous process as an interbody graft between 2010-2014 years were examined. Among them, 51 patients who met the study criteria and could be followed up thereby classified as group A. In this group, spondylolisthesis was present at the L4/L5 level in 34 patients and at the L5/S1 level in 17 patients. In this group, 34 patients had spondylolisthesis at L4/L5 level and 17 at L5/S1 level.

Group B: Of the 54 patients who underwent PLIF surgery using a synthetic solid calcium hydroxyapatite block (Nanostim,
Medtronic, Memphis, USA), 51were randomly selected and included as the control group. Among them, 32 had spondylolisthesis at L4/L5 level and 19 at L5/S1 level. Each group received approximately equal graft volumes $(8-10 \mathrm{ml})$.

\section{Surgical Technique}

All patients were administered 2 gram of cefazolin 30 minutes preoperatively. Patients were placed on an appropriate spinal frame in the prone position under general anesthesia. Then, a routine posterior midline incision was made. Bilateral paravertebral muscles were laterally retracted to the outer edge of the facet joint, exposing the spinous process and both laminae. In group A, the spinous process was removed via an en bloc resection (Figure 1). After cleaning the soft tissues, these bone fragments were soaked in an antibiotic solution (gentamicin, $0.2 \mathrm{~mL}$ of $2 \%$ solution, $20 \mathrm{mg} / \mathrm{mL} / 100 \mathrm{~g}$, Kocak Farma, Istanbul). Extensive discectomy was performed, and the endplate cartilages were politely removed without curettage. Bone fragments were placed in the anterior portion of the interbody space while preserving the neural structures with a retractor. Then, titanium pedicle screws were inserted bilaterally. Connecting rods and a transverse connector between the rods were inserted to fix pedicle screws, and intraoperative radiography was performed to assess the final position of the metalwork. Meanwhile, in group B, a synthetic solid calcium hydroxyapatite blockgraft (Figure 2) (Surgiwear LTD, Shahjahanpur, India), i.e., a mixture of calcium carbonate and dicalcium phosphate, was soaked in the patient's blood and then in an antibiotic solution (gentamicin, $0.2 \mathrm{~mL}$ of $2 \%$ solution $(20 \mathrm{mg} / \mathrm{mL}) / 100 \mathrm{~g}$, Kocak Farma, Istanbul). Thereafter, the graft was placed at the anterior portion of the interbody space while preserving the neural structures with a retractor. Titanium pedicle screws were inserted bilaterally. Connecting rods and a transverse connector between the rods were inserted to fix the pedicle screws, and intraoperative radiography was performed to assess the final position of the metalwork.

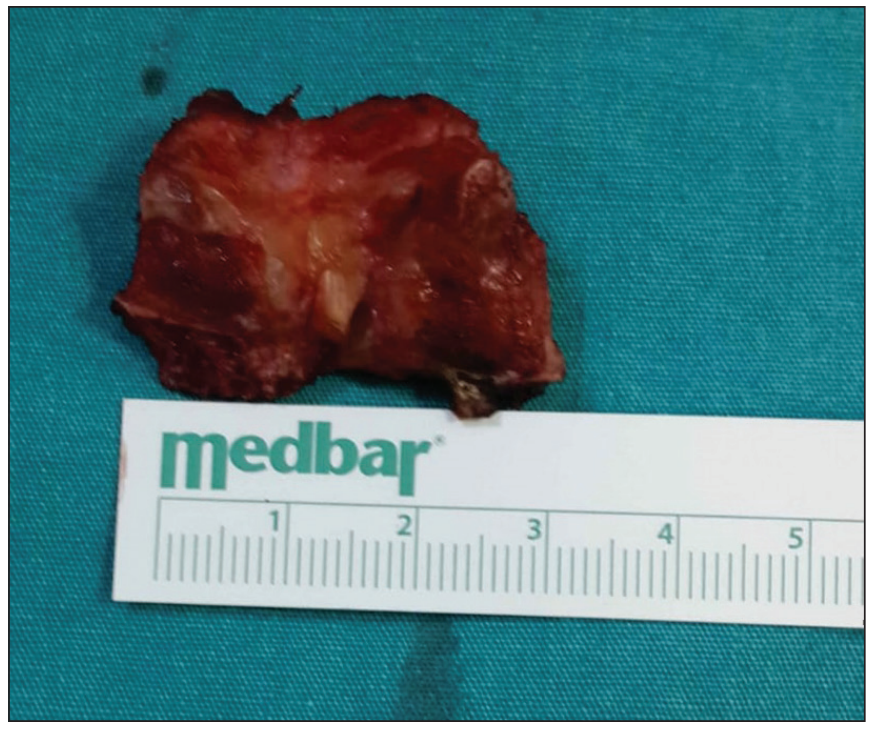

Figure 1: An autograft obtained from the spinous process during total decompression. 


\section{Analysis Method}

Preoperatively; age, sex, body mass index (BMI), presence of comorbidities such as diabetes mellitus, thyroid disorder, and other endocrine diseases, psychiatric disorders requiring drug use, and bone mineral density of the femoral neck examined using dual-energy X-ray absorptiometry (Osteoprima, Mediray, Suwon, Korea) were compared between the two groups. To measure patients' clinical and pain outcomes, the Oswestry Disability Index (ODI) and visual analog scale (VAS) scores were recorded preoperatively and at 6 months and 5 years postoperatively.

The degree of bone formation at the operative level was assessed and compared between the two groups using a computed tomography sagittal reconstruction image according to the method used by Ahn et al. (1). Scores for the degree of bone formation were as follows: grade 1, lack of bone formation; grade 2 , bone formation in $\leq 50 \%$ of the grafted zone; grade 3, bone formation in $>50 \%$ of the grafted zone; and grade 4, diffuse and continuous bone formation. In addition, intervertebral disc heights from the middle of the superior border to the inferior border were measured (Figure 3), and compared between the groups. In addition, complications such as instrument failure and graft subsidence were recorded.

Informed consent forms were obtained from all patients, and this study was approved by the Ethics Committee of Erzincan Binali Yildirim University Medical School (2016; 42-17).

\section{Statistical Analysis}

Results are presented as numbers and percentages for categorical variables and as mean (minimum-maximum) for continuous variables. Categorical variables were compared using the chi-square or Fisher's exact test. The significance level for all analyses was accepted at 0.05. Statistical analysis was performed using the Statistical Package for the Social Sciences software version 19.0 (released in 2010; IBM Corp., Armonk, NY, USA). And the maximal information coefficient (MIC) statistical method was used for the detection of linear and non-linear relationships between continuous variables. To calculate MIC values "minerva" package in $\mathrm{R}$ ver 3.5.3 was used. MIC takes values from 0 to 1 ; the closer it is to 1 , the stronger relationship between the variables was expected.

\section{RESULTS}

No significant difference in age, sex, BMI, presence of medical comorbidities, psychiatric disorders, and bone mineral density values was observed between the two groups (Table I).

A significant improvement in ODI scores was observed in both groups at 6 months and 5 years postoperatively $(p<0.05$ and $p<0.05$, respectively), without significant difference when compared ( $p=0.775$ and $p=0.351$, respectively; Table II).

VAS scores were significantly improved in both groups at 6 months and 5 years postoperatively $(p<0.05$ and $p<0.05$, respectively), but without significant difference when compared ( $p=0.541$ and $p=0.132$, respectively; Table III).
Adequate bone formation was observed in both groups at 6 months and 5 years postoperatively, even in group A (Figure 4). However, no significant difference was found when compared (Table IV).

And according to the MIC values there were no linear or non linear relationships were found between paired variables.

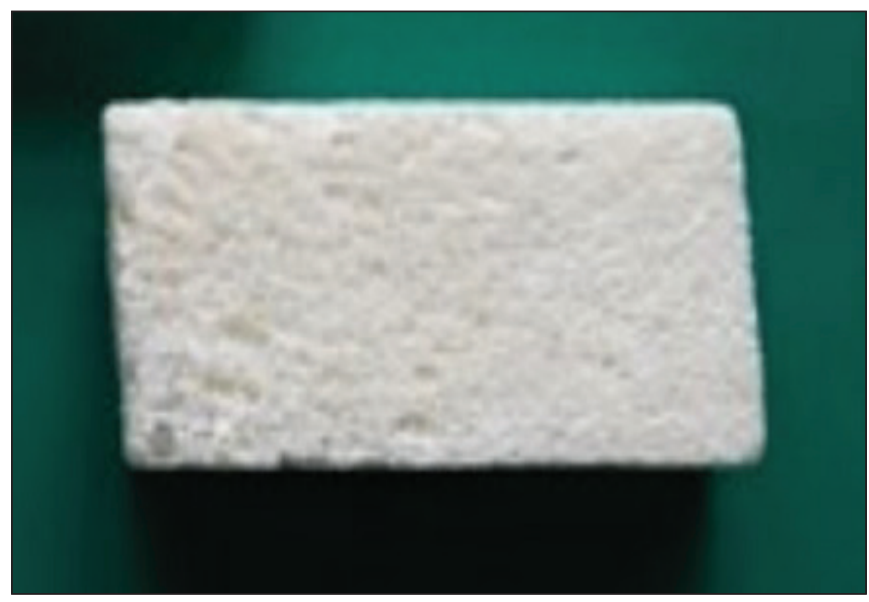

Figure 2: A synthetic solid calcium hydroxyapatite blockgraft.

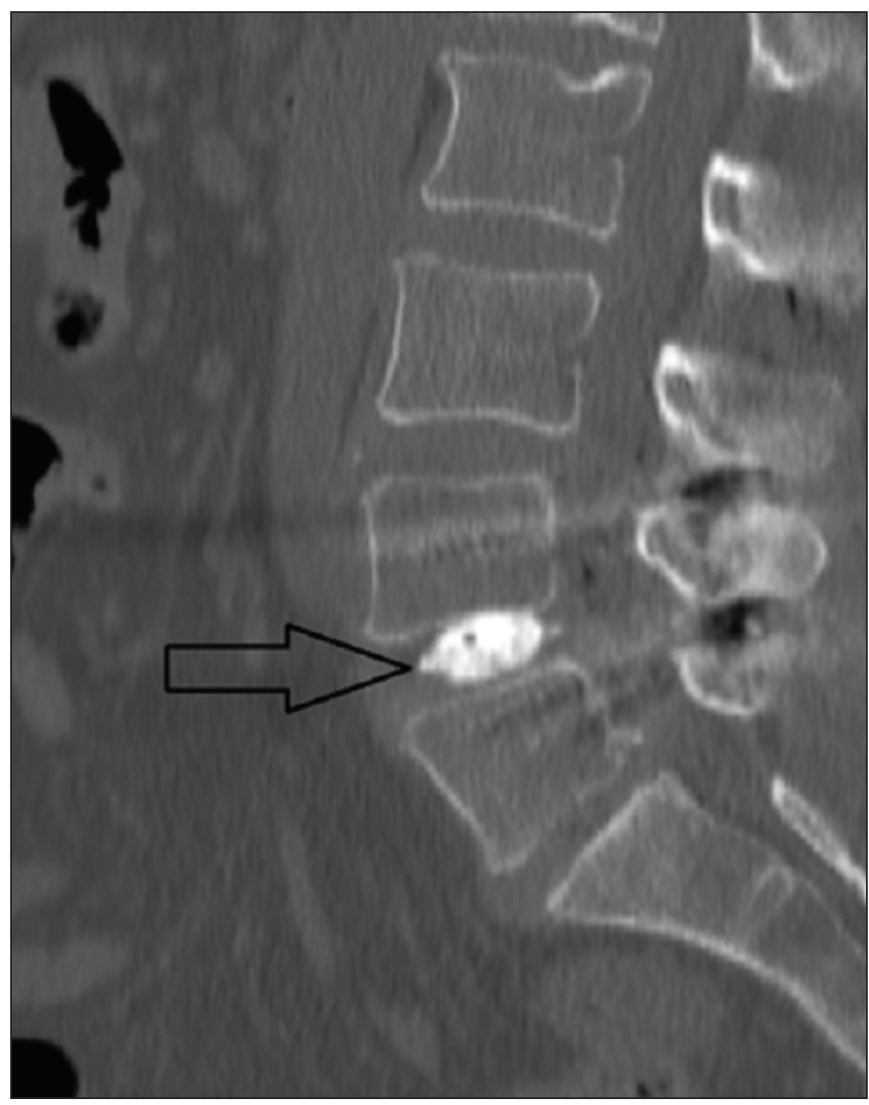

Figure 3: The large arrow indicates the synthetic bone graft. The degree of bone formation is grade 2; notably, fusion at the end plates has not advanced. 
Table I: Characteristics of the Study Patients

\begin{tabular}{lccc}
\hline & $\begin{array}{c}\text { Group A } \\
\mathbf{n = 5 1}\end{array}$ & $\begin{array}{c}\text { Group B } \\
\mathbf{n = 5 1}\end{array}$ & $\mathbf{p}$ \\
\hline Age (range) & $66.1(39-71)$ & $61.4(37-67)$ & 0.447 \\
\hline Sex (Male/Female) & $33 / 18$ & $29 / 22$ & 0.440 \\
\hline Body Mass Index & 25.9 & 28.2 & 0.090 \\
\hline Smoking & 24 patients (47\%) & 19 paitents (38\%) & 0.619 \\
\hline Diabetes Mellitus & 13 patients (26\%) & 14 patients (28\%) & 0.790 \\
\hline Other hormone disorders & 3 patients (7\%) & 2 patients (4.7\%) & 0.910 \\
\hline Psychiatric illness & 5 patients (9.5\%) & patients (16.6\%) & 0.107 \\
\hline Bone mineral density & $-1.7 \pm 0.4$ & $-1.9 \pm 0.9$ & 0.353 \\
\hline Operation level (L4-L5/L5-S1) & $34 / 17$ & $32 / 19$ & 0.545 \\
\hline
\end{tabular}

Table II: Functional Improvement in the Two Groups

\begin{tabular}{lccc}
\hline & Preoperative ODI & ODI (6 & ODI (5 $^{\text {th }}$ year) \\
\hline Group A & $61.7 \pm 4.4$ & $34.1 \pm 1.4$ & $39.5 \pm 7.1$ \\
\hline Group B & $60.8 \pm 8.0$ & $37.4 \pm 4.3$ & $38.5 \pm 3.8$ \\
\hline $\mathbf{p}$ & & 0.775 & 0.351 \\
\hline
\end{tabular}

Table III: Visual Analog Scale Scores of the Two Groups

\begin{tabular}{lccc}
\hline & Preoperative VAS & VAS (6 ${ }^{\text {th }}$ month) & VAS (5 ${ }^{\text {th }}$ year) \\
\hline Group A & $9.3 \pm 1.0$ & $3.1 \pm 1.4$ & $4.3 \pm 0.2$ \\
\hline Group B & $8 \pm 1.1$ & $3.6 \pm 0.3$ & $3.5 \pm 1.6$ \\
\hline p & & 0.541 & 0.132 \\
\hline
\end{tabular}

Table IV: Comparison of the Bone Formation Scores

\begin{tabular}{lcc}
\hline & $\begin{array}{c}\text { Bone formation } \\
\left(\mathbf{6}^{\text {th }} \text { month) }\right.\end{array}$ & $\begin{array}{c}\text { Bone formation } \\
\left.\mathbf{( 5}^{\text {th }} \text { year }\right)\end{array}$ \\
\hline Group A & $2.7 \pm 1.8$ & $3.4 \pm 0.18$ \\
\hline Group B & $2.01 \pm 1.1$ & $2.1 \pm 3.12$ \\
\hline p & 0.140 & 0.092 \\
\hline
\end{tabular}

In group $A$, no significant correlation was found between improvement of VAS in both 6th month and 5th years with bone formation values $(\mathrm{MIC}=0.045$ and $\mathrm{MIC}=0.132$, respectively). Similarly, in group $B$, there was no significant correlation between improvement of VAS in both $6^{\text {th }}$ month and $5^{\text {th }}$ years with bone formation values ( $\mathrm{MIC}=0.017$ and $\mathrm{MIC}=0.038$, respectively).

Moreover, both groups did not significantly differ in terms of intervertebral disc height protection (Table V). In group A, three patients required second surgery due to screw malposition in the early period and were discharged with normal neurological

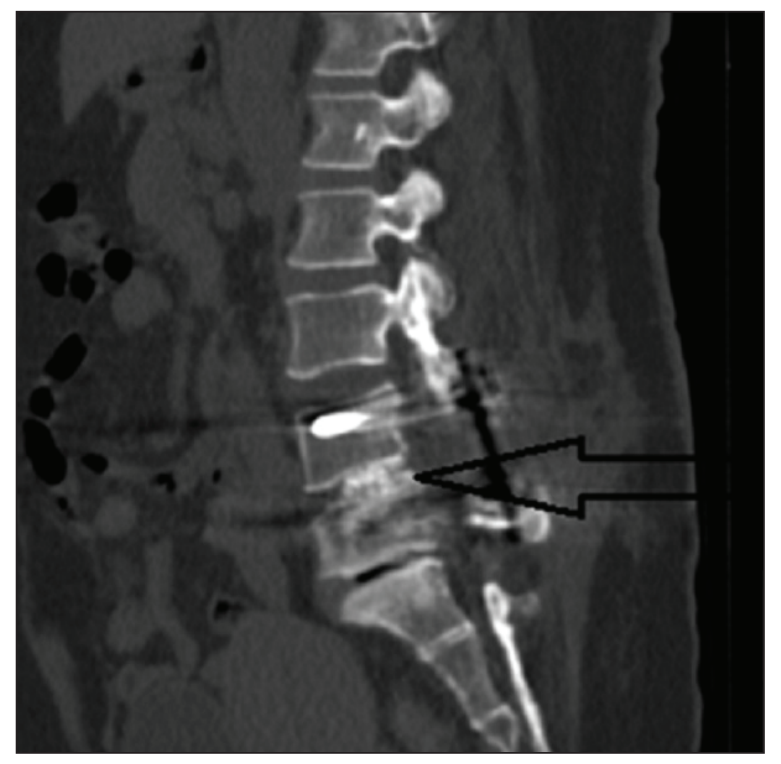

Figure 4: Computed tomography image of a patient in group $A$ obtained at postoperative 5 years. The black arrow indicates the interbody fusion (grade 4; diffuse and continuous bone formation). 
Cakir T. and Yolas C: Synthetic Bone Graft versus Autograft

Table V: Comparison of the Intervertebral Disc Heights

\begin{tabular}{lccc}
\hline & Preoperative disc height $(\mathbf{m m})$ & Disc height $\mathbf{6}^{\text {th }}$ month) $(\mathbf{m m})$ & Disc height $\left(\mathbf{5}^{\text {th }}\right.$ year) $(\mathbf{m m})$ \\
\hline Group A & $9.23 \pm 2.3$ & $8.1 \pm 0.9$ & $7.1 \pm 0.88$ \\
\hline Group B & $10.1 \pm 1.2$ & $7.91 \pm 1.7$ & $7.0 \pm 2.1$ \\
\hline p & & 0.221 & 0.561 \\
\hline
\end{tabular}

examination results, whereas in group $\mathrm{B}$, only one patient required the second surgery. Rod fracture occurred in three patients in group A during the 5-year follow-up, whereas no rod fracture occurred in group $B$, but without significant difference when compared $(p=0.24)$. In addition, no graft subsidence was observed in both groups duringthe5-year follow-up. In group A, one patient $(2.4 \%)$ had a surgical site infection, with Escherichia coli found during bacterial culture. The patient recovered with a medical treatment, without requiring a second surgery.

In group B, surgical site infections occurred in six (14.2\%) patients. The growth of Staphylococcus aureus and E. coli was observed in cultures of 4 and 1 patient, respectively. The cause of infection in one patient could not be identified. All of them responded to medical treatments, without requiring a second surgery. However, in group B, spondylodiscitis at the operation level was observed in one patient at 1year postoperatively. This patient was hospitalized, administered with IV antibiotic therapy, and was discharged with healing. He was closely followed up for infection recurrence. Lastly, no significant difference in the incidence of surgical site infections was observed between the two groups $(p=0.109)$.

\section{DISCUSSION}

In addition to posterior transpedicular instrumented fusion, PLIF has been considered as an effective surgical technique for degenerative disc disease and spondylolisthesis $(20,28)$. Previous studies demonstrated that successful fusion of an unstable segment in degenerative diseases plays a significant role inthepatient's clinical improvement $(4,15,18,20,28)$. This technique remains the suggested standard treatment to date due to its simultaneous neural structure decompression and positive effect on the fusion $(4,5,14,15)$. Several studies reported that a successful sagittal balance can be achieved with PLIF $(9,15)$, which is undoubtedly beneficial in relieving postoperative back pain and accelerating the postoperative rehabilitation in patients who undergo degenerative spondylolisthesis surgery (9). In the PLIF surgery, the use of an appropriate graft to induce ossification is as important as the surgical method in achieving a successful interbody fusion $(3,19,20,23)$. Several studies indicated that the ideal graft for PLIF is one that will cause the least donor-site morbidity and provide a maximum bone growth efficacy by combining osteoinduction, osteoconduction, and osteoblastic properties (20). In this context, a cage or a bone graft obtained from the iliac crest reported by Cloward in the 1950s remains to be commonly used to date (8). Although complications such as dural rupture and cage subsidence may occur, intervertebral cages have been reported to facilitatea successful fusion in approximately $90-95 \%$ of patients (14). Furthermore, adequate interbody fusion can be achieved using an autograft obtained from the iliac crest. However, some complications such as prolonged operation time, blood loss, persistent donor site pain reported by approximately $30 \%$ of patients, and infection may occur (10). In the literature, the number of studies on the use of the spinous process as an interbody graftis limited. In addition, successful results were obtained in our study. For example, good bone formation, successful fusion, significantly decreased back pain, and adequate protection of the intervertebral disc space were observed at the end of the 5-year follow-up. Hu et al. reported that 36 PLIF procedures were performed using the patient's lumbar spinous process as a bone graft without using a cage and showed sufficient interbody fusion at the motion segment and clinically successful patient outcomes (14). Moreover, they particularly reported that soft tissues should be thoroughly cleaned during the graft preparation, and the graft should be placed at the front of the intervertebral area as much as possible. Lin et al. also achieved a successful interbody fusion rate while performing PLIF surgery using an autograft obtained from the spinous process (20). These studies showed that using bone fragments obtained from the patient him-/herself instead of synthetic grafts seems more logical to provide better ossification. Using a synthetic implant in the intervertebral disc space may reduce the contiguity ossification in the fusion area because experimental studies demonstrated that $>30 \%$ of the surface area of the end plate should be in direct contact with the local bone during an interbody fusion (6).

In addition, the difference in complication rates was not significant between the two groups. For example, there was no graft breakage or subsidence in both groups. And the intervertebral disc spaces were adequately preserved in both groups. Probably politely removing the endplates without curettage and preserving the intervertebral disc distance with the help of the posterior transpedicular stabilization caused no graft breakage or subsidence. In the literature, it has been mentioned that posterior transpedicular stabilization maintains the height of the intervertebral disc space, prevents spinal instability, and therefore prevents complications associated with graft as much as possible $(21,22)$. Also the rate of post operative infection was much lower in the group we used spinous process graft. And this was compatible with studies in the literature. For example, Hu et al. found a postoperative infection rate of $2.6 \%$ (14). However, our study found that using a graft obtained from the patient's own bone tissues results in virtually no potential risk of foreign body reaction. Finally, this procedure reduces the cost of surgery, which 
could be especially appealing in developing countries with fewer resources. As patients who used spinous process grafts did not require additional treatment cost, approximately 60100 euros is additionally spent when using synthetic grafts, depending on its size.

\section{CONCLUSION}

This study demonstrated that a satisfactory fusion rate can be achieved and the intervertebral height restored and maintained after performing a PLIF using a natural graft obtained from the patient's spinous process. These results suggest that a natural graft is effective and safe and can be considered as a cost-effective alternative method especially for developing countries.

\section{REFERENCES}

1. Ahn DK, Moon SH, Kim TW, Boo KH, Hong SW: Demineralized bone matrix, as a graft enhancer of auto-local bone in posterior lumbar interbody fusion. Asian Spine Journal 8(2):129, 2014

2. Alfieri A, Gazzeri R, Prell J, Röllinghoff M: The current management of lumbar spondylolisthesis. Journal of Neurosurgical Sciences 57(2):103-113, 2013

3. Bostrom MPG, Seigerman DA: The clinical use of allografts, demineralized bone matrices, synthetic bone graft substitutes and osteoinductive growth factors: A survey study. HSS J 1(1):9-18, 2005

4. Cakir T, Cakir M, Okay HO, Yolas C, Tanriverdi O, Omeroglu $\mathrm{M}$, Arslan YK: Single level discectomy with and without disc prosthesis: A comparative study of 114 patients. Medicine 97(52):e13854, 2018

5. Christensen FB, Hansen ES, Eiskjaer SP, Hoy K, Helmig P, Neumann P, Niedermann B, Bunger CE: Circumferential lumbar spinal fusion with Brantigan cage versus posterolateral fusion with titanium Cotrel-Dubousset instrumentation: A prospective, randomized clinical study of 146 patients. Spine (Phila Pa 1976) 27(23):2674-2683, 2002

6. Closkey RF, Parsons JR, Lee CK, Blacksin MF, Zimmerman MC: Mechanics of interbody spinal fusion. Analysis of critical bone graft area. Spine 18(8):1011-1015, 1993

7. Cloward RB: Spondylolisthesis: Treatment by laminectomy and posterior interbody fusion. Review of 100 cases. Clin Orthop 154:74-82, 1981

8. Cloward RB: The treatment of ruptured lumbar intervertebral discs by vertebral body fusion: Indication, operative technique, after care. J Neurosurg 10:154-168, 1953

9. Cunningham JE, Elling EM, Milton $A H$, Robertson PA: What is the optimum fusion technique for adult isthmic spondylolisthesis-PLIF or PLF? A long-term prospective cohort comparison study. Clinical Spine Surgery 26(5):260267, 2013

10. France JC, Schuster JM, Moran K, Dettori JR: Iliac crest bone graft in lumbar fusion: The effectiveness and safety compared with local bone graft, and graft site morbidity comparing a single-incision midline approach with a two-incision traditional approach. Global Spine Journal 28(03):195-206, 2015
11. Guigui P, Ferrero E: Surgical treatment of degenerative spondylolisthesis. Orthopaedics \& Traumatology: Surgery \& Research, 103(1):S11-S20, 2017

12. Hayashi K, Matsumura A, Konishi S, Kato M, Namikawa T, Nakamura $\mathrm{H}$ : Clinical outcomes of posterior lumbar interbody fusion for patients 80 years of age and older with lumbar degenerative disease: Minimum 2 years' follow-up. Global Spine Journal 6(07):665-672, 2016

13. Høy K, Bünger C, Niederman B, Helmig P, Hansen ES, Li $\mathrm{H}$, Andersen $\mathrm{T}$ : Transforaminal lumbar interbody fusion (TLIF) versus posterolateral instrumented fusion (PLF) in degenerative lumbar disorders: A randomized clinical trial with 2-year follow-up. European Spine Journal 22(9):2022-2029, 2013

14. Hu MW, Liu ZL, Zhou Y, Shu YL, Chen C, Yuan X: Posterior lumbar interbody fusion using spinous process and laminae. J Bone Joint Surg Br 94(3):373-377, 2012

15. Kai Y, Oyama M, Morooka M: Posterior lumbar interbody fusion using local facet joint autograft and pedicle screw fixation. Spine 29(1):41-46, 2004

16. Kim K, Youn Y, Lee S H, Choi JC, Jung J E, Kim J, Kim T $\mathrm{H}$ : The effectiveness and safety of nonsurgical integrative interventions for symptomatic lumbar spinal spondylolisthesis: A randomized controlled multinational, multicenter trial protocol. Medicine 97(19):e0667, 2018

17. Kunder SL, van Kuijk SM, Rijkers K, Caelers IJ, van Hemert WL, de Bie RA, van Santbrink H: Transforaminal lumbar interbody fusion (TLIF) versus posterior lumbar interbody fusion (PLIF) in lumbar spondylolisthesis: A systematic review and meta-analysis. Spine Journal 17(11):1712-1721, 2017

18. Lee KJ, Roper JG, Wang JC: Demineralized bone matrix and spinal arthrodesis. Spine J 5 Suppl:217S-23S, 2005

19. Lementowski PW, Lucas P, Taddonio RF: Acute and chronic complications of intracortical iliac crest bone grafting versus the traditional corticocancellous technique for spinal fusion surgery. Orthopedics 33(4):1429-1435, 2010

20. Lin B, Yu H, Chen Z, Huang Z, Zhang W: Comparison of the PEEK cage and an autologous cage made from the lumbar spinous process and laminae in posterior lumbar interbody fusion. BMC Musculoskeletal Disorders 17(1):374, 2016

21. Luque $\mathrm{E}:$ The anatomical basis and development of segmental spinal instrumentation. Spine 7:256-259, 1982

22. Panjabi M, Abumi K, Durancean J, Crisco J: Biomechanicl evaluation of spinal fixation devices - stability provided by eight internal fixation devices. Spine 13:1135-1140, 1988

23. Ploumis A, Albert TJ, Brown Z, Mehbod AA, Transfeldt EE: Healos graft carrier with bone marrow aspirate instead of allograft as adjunct to local autograft for posterolateral fusion in degenerative lumbar scoliosis: A minimum 2-year follow-up study. J Neurosurg Spine 13(2):211-215, 2010

24. Scholz M, Schleicher P, Kandziora F: Instrumented lumbar interbody fusion L4-S1 (TLIF L4-S1). European Spine Journal 26(3):416-417, 2017

25. Tropiano P, Huang RC, Girardi FP: Lumbar disc replacement: Preliminary results with Prodisc2 after a minimum follow-up period of 1 year. Clinical Spine 16:362-368, 2003 
26. Vaz K, Verma K, Protopsaltis T, Schwab F, Lonner B, Errico T: Bone grafting options for lumbar spine surgery: A review examining clinical efficacy and complications. International Journal of Spine Surgery 4(3):75-86, 2010

27. Weatherley CR, Prickett CF, O'brien JP: Discogenic pain persisting despite solid posterior fusion. J Bone Joint Surg $\mathrm{Br}$ 68(1):142-143, 1986
28. Wetzel FT, Brustein M, Phillips FM, Trott S: Hardware failure in an unconstrained lumbar pedicle screw system. A 2-year follow-up study. Spine (Phila Pa 1976) 24(11):1138-1143, 1999

29. Zhou ZJ, Xia P, Zhao X, Fang XQ, Zhao FD, Fan SW: Can posterior dynamic stabilization reduce the risk of adjacent segment deterioration? Turk Neurosur 23(5):579-589, 2013 